\title{
From data to science: Planck data and the CMB non-Gaussianity
}

\author{
Anna Mangilli ${ }^{1}$ on behalf of the Planck collaboration \\ ${ }^{1}$ Institut d'Astrophysique de Paris, CNRS and UPMC Sorbonne Universites Paris VI, \\ Bd. Arago , 75014, Paris, France \\ email: mangilli@iap.fr
}

\begin{abstract}
Studying the non-Gaussianity (NG) of the Cosmic Microwave Background (CMB) is an extremely powerful tool to investigate the properties of the very early Universe. The Planck nominal mission CMB maps yielded unprecedented constraints on primordial non-Gaussianity providing with the highest precision test of the standard model of inflation. Planck's high sensitivity also allowed to find evidence for the first time of the late-time non-Gaussianity arising from the Lensing-Integrated Sachs Wolfe (ISW) cross correlation. In this talk I will give details on the Planck data analysis and I will discuss the theoretical implications of the results.
\end{abstract}

Keywords. (cosmology:) cosmic microwave background, methods: data analysis, cosmology: observations, (cosmology:) early universe

\section{Overview}

One of the main reasons of the popularity of the standard inflationary model relies on the fact that inflation provides a mechanism that can explain the production of the first density perturbations in the early Universe which are the seeds of the large scale structure in the distribution of galaxies and of the Cosmic Microwave Background (CMB) temperature anisotropies that we observe today. Studying the CMB non-Gaussianity, i.e. the statistical properties of the $\mathrm{CMB}$ anisotropies beyond the linear regime, emerged as one of the highest precision test for the standard inflationary framework as a key prediction of the simplest models of inflation is a very small level of CMB non-Gaussianity (Acquaviva et al. (2003); Maldacena (2003)). In general the CMB non-Gaussianity is related to non-linear mechanisms and it can be roughly divided into two main categories:

- Primordial type non-Gaussianity: refers to the CMB non-Gaussianity due to the non-linear processes happening between the end of inflation (or equivalent mechanism) and the last scattering surface. A detection of the primordial type non-Gaussianity in the CMB would rule out the standard single field slow roll inflation and would be the smoking gun of a different scenario for the generation of the primordial perturbations. Constraining primordial non-Gaussianity (NG) is fully complementary e.g. to looking for the imprint of the primordial gravity waves in the B-modes CMB polarization. An example: the local type non-Gaussainity. One of the most common example of primordial NG is given by the so called local type, where the non-Gaussianity arises because of the non-linear corrections to the gravitational potential's perturbations $\Phi(\mathbf{x})$, parametrized by $f_{N L}$, the amplitude of the quadratic non-linear contribution (Salopek \& Bond (1990); Gangui et al.(1994); Verde et al. (2000); Komatsu \& Spergel (2001)):

$$
\Phi(\mathbf{x})=\Phi_{L}(\mathbf{x})+f_{N L}\left(\Phi_{L}^{2}(\mathbf{x})-\left\langle\Phi_{L}^{2}(\mathbf{x})\right\rangle\right)
$$

This type of non-Gaussianity is very small in the case of the standard slow roll inflation but can be big for alternatives models as for example multi field inflationary models. 
- Late-time type non-Gaussianity: refers to the CMB non-Gaussianity that arises because of the non-linear processes in the late time stage of the Universe evolution when the structures form. The lensing-ISW late-time NG: an extremely interesting late time non-Gaussian signal arises in the CMB because of the correlation of two effects: the lensing and the Integrated Sachs Wolfe (ISW) effect. Weak lensing is responsable for the deflection of the CMB photons trajectories when they pass by the gravitational potential wells of the structures, while the ISW effect is related to the fact that in the late time evolution, when dark energy starts dominating $(z<2)$, the gravitational potentials are "stretched" by the accelerated expansion causing a net gain of energy for the CMB photons that climb out these less and less deep overdensities. Both lensing and the ISW effect are related to the same gravitational potentials distribution, lensing by itself is a non-linear and non-Gaussian process, therefore the combination of these two effects gives rise to the lensing-ISW non-Gaussian signal in the CMB. This signal is extremely interesting because it provides with a new direct probe of the action of dark energy on the evolution of the structures. Also, it has been shown that the lensing-ISW nonGaussianity, if not properly accounted for, can be a serious contaminant for the estimation of the primordial local signal, so it is extremely important to correctly model it.

\subsection{The CMB bispectrum}

In the case of gaussian perturbations the CMB power spectrum encloses all the statistical information. On the other hand, even a small deviation from gaussianity would imply that the CMB sky encodes more informations and in order to access them it is necessary to study the higher order statistics of the CMB anisotropies beyond the power spectrum. The CMB three-points function, or its spherical harmonic projection called the CMB angular bispectrum, is the best candidate preposed for this task being the lowest order non-gaussian statistic and in principle the most easily detectable. The CMB bispectrum is proportional to the non-linear amplitude parameter $f_{N L}$ and it is characterized by a shape function. There are many different (triangle) shapes that can be formed and, more importantly, different shapes are related to different phenomena. There are three main fundamental CMB bispectrum shapes motivated by different classes of models:

Squeezed-local bispectrum shape: this shape is very important because it is the shape of the local type non-Gaussianity mentioned in Sec. 1 and it is motivated e.g. by multi-fields inflationary models or alternatives like the ekpirotic/cyclic models. Also, the squeezed shape characterizes the late-time lensing-ISW bispectrum. This shape degeneracy explains why the lensing-ISW bispectrum can contaminate the estimation of the primordial local bispectrum amplitude with a bias on $f_{N L}^{\text {local }}$ that has been estimated to be $\Delta f_{N L} \simeq 10$ (Mangilli \& Verde (2009); Hanson et al. (2009)). Considering that the errors on $f_{N L}^{\text {local }}$ from Planck and future CMB experiments are $\sigma_{f_{N L}^{\text {local }}}<10$, the lensing-ISW signal must be correctly included in the analysis in order to get unbiased estimates of the local type primordial non-Gaussianity. Equilateral bispectrum shape: this shape is motivated by classes of inflationary models as for example the DBI and ghost inflation or in general models with e.g. non-canonical kinetic terms. Orthogonal bispectrum shape: also this shape is motivated by inflationary models with a more complicated inflationary dynamics with higher order derivatives and/or non-canonical kinetic terms in the inflaton's action.

\subsubsection{Optimal estimators for $f_{N L}$}

One of the main goal of the CMB non-Gaussian analysis is to constrain with high accuracy the amplitude parameter $f_{N L}$ for different bispectrum shapes. In order to do so, an optimal bispectrum estimator for $f_{N L}$ can be constructed. This is based on a 

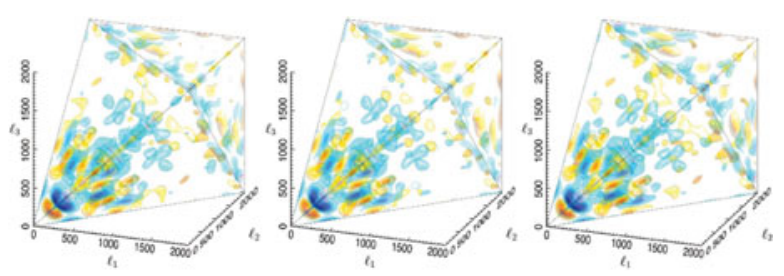

Figure 1. The Planck bispectrum reconstruction for three different component separation methods: SMICA, SEVEM, NILC (The Planck collaboration XXIV (2013)).

least-squares fit comparison of the observed CMB bispectrum with a given theoretical bispectrum template $b_{\ell_{1} \ell_{2} \ell_{3}}^{t h}$ and takes the form:

$$
\begin{aligned}
\hat{f}_{N L} & \equiv \frac{1}{N} \sum_{\text {all lm }} \mathcal{G}_{l_{1} l_{2} l_{3}}^{m_{1} m_{2} m_{3}} b_{\ell_{1} \ell_{2} \ell_{3}}^{t h}\left[\left(C^{-1} a\right)_{\ell_{1} m_{1}}\left(C^{-1} a\right)_{\ell_{2} m_{2}}\left(C^{-1} a\right)_{\ell_{3} m_{3}}\right. \\
& \left.-3\left(C^{-1}\right)_{\ell_{1} m_{1}, \ell_{2} m_{2}}\left(C^{-1} a\right)_{\ell_{3} m_{3}}\right] .
\end{aligned}
$$

The linear term $\hat{f}_{N L}^{\text {linear }}=-3\left(C^{-1}\right)_{\ell_{1} m_{1}, \ell_{2} m_{2}}\left(C^{-1} a\right)_{\ell_{3} m_{3}}$ is needed in the case of a realistic experimental setting in order to correct for spurious terms due to e.g. the anisotropic noise and/or the incomplete sky cuts when applying a mask. Different types of optimal estimators can be defined depending on the properties of the theoretical bispectrum templates.

\section{Planck data analysis and results}

The results presented here are a summary of the Planck 2013 results detailed in: The Planck collaboration XXIV (2013); The Planck collaboration XIX (2013); The Planck collaboration XVII (2013); The Planck collaboration XXIII (2013).

Planck $\dagger$ is the ESA telescope that observed the sky in 9 frequency bands from 30 to $857 \mathrm{GHz}$ with an unprecedented sensitivity, opening for the first time the possibility to constrain the CMB non-Gaussianity with very high precision. In order to constrain the CMB bispectrum the cleanest as possible CMB map is needed so that the Planck data have been processed through the component separation pipelines in order to correctly remove the foregrounds contamination. Fig. 1 presents the Planck bispectrum reconstruction in the case of three different methods of foregrounds removal (SMICA, SEVEM, NILC), showing that the bispectrum reconstruction is extremely robust with respect to foreground cleaning. In order to constrain the bispectrum amplitude $f_{N L}$, different type of estimators have been used for the Planck data analysis. In particular for the analysis of the primordial bispectrum shapes the main optimal estimators used are: the separable KSW estimator (Komatsu, Spergel \& Wandelt (2005)), the skew- $C_{\ell}$ (Munshi \& Heavens (2009)), the modal (Fergusson et al. (2010)) and binned (Bucher et al. (2010)) estimators. Also, in order to correctly account for lensing-ISW contribution, the following optimal estimators specifically built for the lensing-ISW signal have been used: Mangilli et al.(2013) for the KSW and Lewis et al. (2011) for the lensing reconstruction estimator. All the estimators have been massively tested on realistic Planck FFP6 lensed simulations processed the same as the data and they showed a remarkable map-by-map agreement and consistency. The main results of the Planck data analysis on the constraints of $f_{N L}$ for the three fundamental primordial shapes and for the lensing-ISW bispectrum are reported in Table 1. Planck finds evidence of the squeezed local type non-Gaussianity: as expected

$\dagger$ http://sci.esa.int/planck/ 
Table 1. Summary of the Planck constraints on $f_{N L}$ for the three primordial fundamental shapes and for the lensing-ISW bispectrum (The Planck collaboration XXIV (2013); The Planck collaboration XIX (2013))

\begin{tabular}{cccc}
\hline & KSW & Binned & Modal \\
\hline Local biased lensing-ISW & $9.8 \pm 5.8$ & $9.2 \pm 5.9$ & $8.3 \pm 5.9$ \\
Local unbiased & $2.7 \pm 5.8$ & $2.2 \pm 5.9$ & $1.6 \pm 6$ \\
Lensing-ISW bias & $7.7 \pm 1.5$ & $7.7 \pm 1.6$ & $10 \pm 3$ \\
Lensing-ISW & $0.85 \pm 0.32$ & $1.03 \pm 0.37$ & $0.93 \pm 0.37$ \\
Equilateral & $-37 \pm 75$ & $-20 \pm 73$ & $-20 \pm 77$ \\
Orthogonal & $-46 \pm 39$ & $-39 \pm 41$ & $-36 \pm 41$ \\
\hline
\end{tabular}

this is due to the lensing-ISW bias that is found to be compatible with the theoretical expectations (Mangilli \& Verde(2009), Hanson et al.(2009)). Only after the subtraction of the lensing-ISW contribution the Planck analysis shows that there is no evidence of primordial local type non-Gaussianity. Also, Planck does not find evidence of primordial equilateral and orthogonal non-Gaussianity. This means that the Planck analysis favors the simplest models for inflation that in this way has passed the most stringent test to date. Also, Planck finds evidence for the first time of the late-time lensing-ISW bispectrum, compatible with the signal expected in the $\Lambda$ CDM scenario. In general the Planck data analysis showed a very good internal consistency, stability across frequencies, stability for changes of the masks and proved to be robust with respect to the contamination from noise and foregrounds. The Planck constraints on CMB non-Gaussianity provided with the highest precision test to date of the physical mechanism of the origin of cosmic structure.

\section{References}

The Planck collaboration, Planck 2013 Results XXIV A\&A 2013/21554

The Planck collaboration, Planck 2013 Results XIX A $\& A 2013$ arxiv:1303.5079

The Planck collaboration, Planck 2013 Results XVII A $\& A 2013$ arXiv:1303.5077

The Planck collaboration, Planck 2013 Results XXIII A\&S 2013 arXiv:1303.5083

Acquaviva V., N. Bartolo, S. Matarrese, \& A. Riotto, Nuclear Physics B 667, 119 (2003)

Bucher, M., van Tent, B., \& Carvalho, C. S. 2010, MNRAS, 407, 2193

Fergusson, J. R., Liguori, M., \& Shellard, E. P. S. 2010a, Phys. Rev. D, 82, 023502

Gangui, A., F. Lucchin, S. Matarrese, \& S. Mollerach, Astrophys. J. 430, 447 (1994)

Hanson, D., K. M. Smith, A. Challinor, \& M. Liguori, Physical Review D 80 :083004 (2009)

Komatsu, E., D. Spergel, \& B. Wandelt, Astrophys. J. 634, 14-19 (2005)

Komatsu, E. \& Spergel, D. N. 2001, Phys. Rev. D, 63, 063002

Lewis, A., et al. JCAP, 03(2011)018

Maldacena, J., Journal of High Energy Physics 5, 13 (2003), arXiv:astro-ph/0210603.

Mangilli, A., B. Wandelt, F. Elsner, \& M. Liguori A\&A 555, A82 (2013)

Mangilli, A., L. Verde, Physical Review D 80, 123007 (10/2009)

Munshi, D. \& Heavens, A. 2010, MNRAS, 401, 2406, arXiv:0904.4478

Salopek, D. S. \& J. R. Bond, Phys. Rev. D 42, 3936 (1990).

Verde, L., Wang, L., Heavens, A. F., \& Kamionkowski, M. 2000, MNRAS, 313, 141 